\title{
Temporal location effect of noise burst intensity on auditory evoked potentials
}

\author{
YASUO KUCHINOMACHI \\ Laboratory of Psychophysiology, Industrial Products Research Institute, Tsukuba Science City, Japan
}

\begin{abstract}
This study was conducted to explore the hypothesis that the N1-P2 component of the auditory evoked potential reflects exclusively the stimulus intensity around $30 \mathrm{msec}$ after sound onset rather than the stimulus intensity immediately after sound onset or the simple integrated value of sound energy. In Experiment 1, subjects were presented randomly with three kinds of brief noise bursts which differed in temporal locations of the plateau intensity. The amplitude of the N1-P2 component was significantly larger in the condition in which the plateau intensity appeared $30 \mathrm{msec}$ after the noise onset than it was in the condition in which it appeared immediately after the onset, and it disappeared less than $30 \mathrm{msec}$ after the onset. Furthermore, the results in Experiment 2 suggest that the N1-P2 component was little influenced by the fall time of noise bursts at approximately $40 \mathrm{msec}$ after the onset. These results, taken together, seem to support the hypothesis stated above.
\end{abstract}

It has been suggested that the auditory system acts as a temporal integrator of sound energy (Olsen \& Carhart, 1966; Zwislocki, 1960, 1969). Onishi and Davis (1968) reported that the amplitude of the N1-P2 component of the auditory evoked potentials recorded from the vertex increased in proportion to the sound duration when it was shorter than $30 \mathrm{msec}$. Furthermore, several researchers found that lengthening the sound duration over about $30 \mathrm{msec}$ did not cause any increase in the amplitude of the N1-P2 component (McCandless \& Best, 1966; Picton, Woods, \& Proulx, 1978). These results, taken together, suggest that the vertex potentials reflect the temporal integration of sound energy within about $30 \mathrm{msec}$ after the stimulus onset.

A close examination of Onishi and Davis' (1968) results, however, reveals that their data cannot be consistently interpreted by the view stated above. For instance, the amplitude of the N1-P2 component of the evoked potential to a sound with a 3-msec rise time and a 30-msec plateau duration was not larger than it was to a sound with a $30-\mathrm{msec}$ rise time and a 3-msec plateau duration. If sound energy is simply integrated over the $33 \mathrm{msec}$ after the stimulus onset, the integrated value in the former sound would be over twice as great as that in the latter sound. Therefore, it is difficult to interpret

This investigation was supported in part by a grant of Special Research Project from the Agency of Industrial Science and Technology in Japan. Correspondence and requests for reprints should be sent to Yasuo Kuchinomachi, Laboratory of Psychophysiology, Industrial Products Research Institute, 1-1-4, Yatabemachi Higashi, Tsukuba Science City, Ibaraki, 305, Japan. this result in terms of simple integration of sound energy. Furthermore, the concept of ceiling effect cannot explain the result satisfactorily.

On the other hand, if it is hypothesized that the stimulus intensity around $30 \mathrm{msec}$ after stimulus onset is more effective for evoking the N1-P2 component than is the stimulus intensity immediately after the stimulus onset, Onishi and Davis' data seem to be easily interpreted.

\section{EXPERIMENT 1}

\section{Method}

The subjects were 11 paid male college students between the ages of 18 and 22 years. All subjects reported that they had no hearing difficulty.

The stimuli were bursts of white noise generated by an NF, WF-711 noise generator. The noise bursts were shaped and temporally controlled by a computer and were presented through a speaker placed $1 \mathrm{~m}$ in front of the subject. Figure 1 shows three types of the shaping-envelopes generated with CPU. The C condition in the top row had a plateau duration of $35 \mathrm{msec}$, and the rise and fall times were $5 \mathrm{msec}$. The plateau durations of the FP condition in the middle row and the SP condition in the bottom row were both $10 \mathrm{msec}$, and their rise and fall times were, respectively, 5 and $30 \mathrm{msec}$ for the former condition and 30 and $5 \mathrm{msec}$ for the latter. Hence, the total duration, from the onset to offset of the stimuli, was $45 \mathrm{msec}$ in all conditions. The temporally integrated energy over the entire $45 \mathrm{msec}$ was equal between the FP and SP conditions, but was greater in the C condition than in either the FP condition or the SP condition. The temporal location of the plateau intensity appeared immediately after stimulus onset in the FP condition but $30 \mathrm{msec}$ after stimulus onset in the SP condition. The intensity of the plateau was set $81 \mathrm{~dB}$ SPL (re $20 \mu \mathrm{N} / \mathrm{m}^{2}$ ) in all the conditions. The sensation levels (SL) of the three patterns ranged between 65 and $70 \mathrm{~dB}$ for the subjects. No remarkable difference in the SLs 


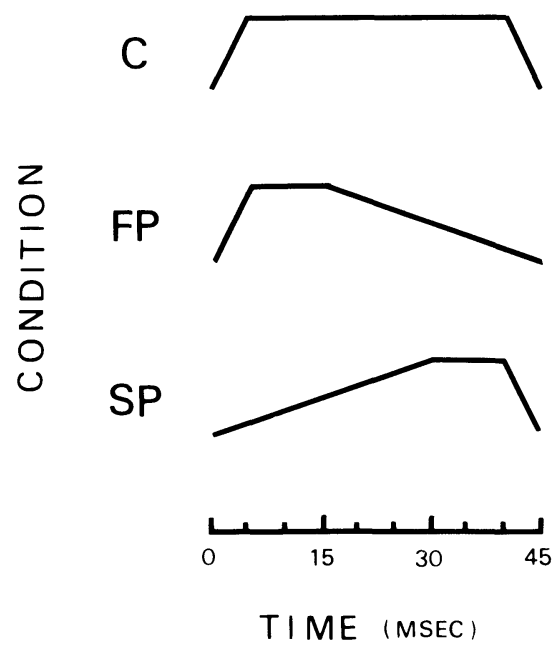

Figure 1. The three types of the shaping-envelopes generated with CPU for noise bursts that differ in the temporal location of the plateau.

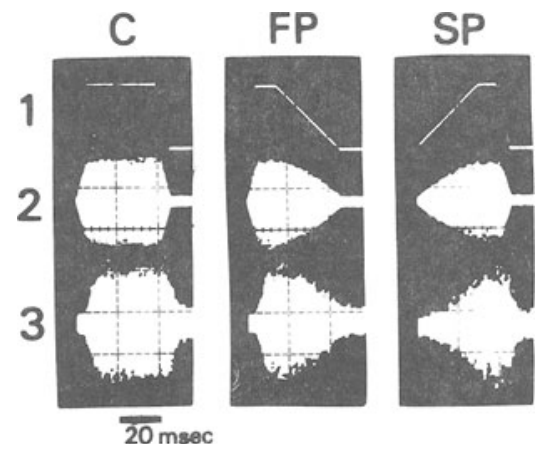

Figure 2. Simultaneous records of shaping-envelopes generated with CPU (1), outputs of multiplier which represent products of the shaping-envelopes and white noise (2), and the speaker's outputs (3), which correspond to three conditions. They are similar in pattern.

existed among the stimulus patterns. Figure 2 shows the patterns of the noise bursts as the speaker's outputs produced by the interaction of the white noise with the shaping-envelopes, as described above. It is clear that outputs of the speaker in the bottom row correspond closely both with their shaping-envelopes in the top row and with the outputs of the multiplier in the middle row.

The subject was given 15 preliminary trials in which he experienced three types of noise bursts five times per type in order to accustom himself to the noise bursts. Then he was presented with three types of noise bursts in random order at a rate of once every $2.5 \mathrm{sec}$. Each type of noise burst was presented 50 times. During the experiment, the subject was seated on a chair in an electrically shielded, quiet, sound-treated room. He was instructed to relax and listen to noise bursts.

Brain potentials were picked up with $\mathrm{Ag}-\mathrm{AgCl}$ electrodes placed at the vertex and the left earlobe. A ground electrode was attached to the right earlobe. A vertical electrooculogram (EOG) was recorded from between the upper and lower orbital ridges of the left eye. These electrical activities were recorded on FM tape (Teac, R-280) for off-line analysis after they were amplified using preamplifiers in which low and high frequency filters were set at a cutoff of .5 and $30 \mathrm{~Hz}$, respectively. Brain potentials in 35 trials were averaged separately for each condition with CPU over an analysis time of $500 \mathrm{msec}$, because the first 10 trials and the trial contaminated by excessive eye activity, as determined from the EOG, were excluded from the averages. The averaged evoked potentials were measured at two peaks: the N1 peak, which was defined as the maximum negativity between 90 and $120 \mathrm{msec}$, and the P2 peak, which was defined as the maximum positivity between 170 and $220 \mathrm{msec}$.

\section{Result}

Peak latencies and amplitudes were analyzed with regard to the three conditions. Amplitude measures were based on the vertical distance between the negative and positive peaks. Figure 3 shows the typical patterns of the evoked potentials of three subjects. No clear trends were apparent in the peak latencies, which did not differ significantly among the conditions. The differences in the amplitudes, however, were clearly observed among the three conditions. Figure 4 shows the mean amplitudes of the N1-P2 components in the evoked potentials of 11 subjects under the three conditions. Obviously, the $\mathrm{C}$ condition has the maximum amplitude, while the FP condition has the minimum value. A one-factor analysis of variance with repeated measurements yielded a significant effect $[F(2,20)=8.73, p<.01]$. Student's

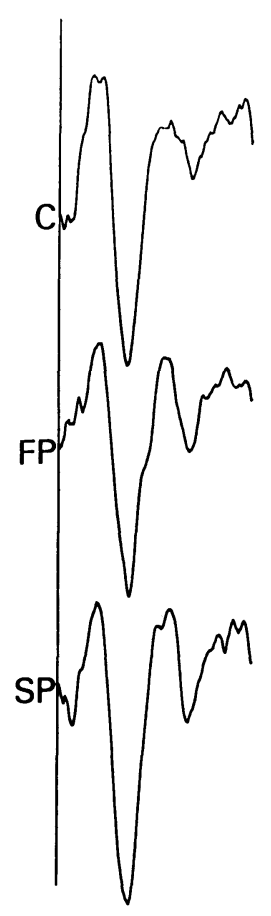

a

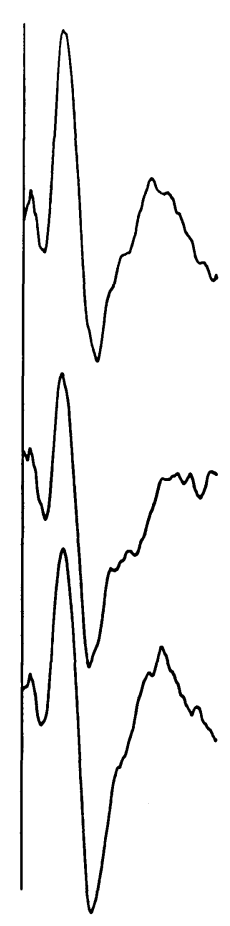

b

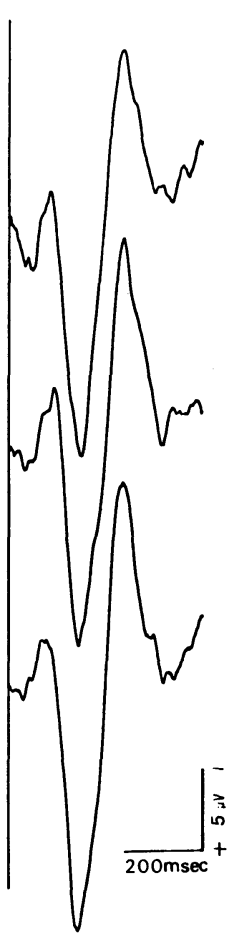

C
Figure 3. The typical auditory evoked potentials of three subjects under three conditions in Experiment 1. 


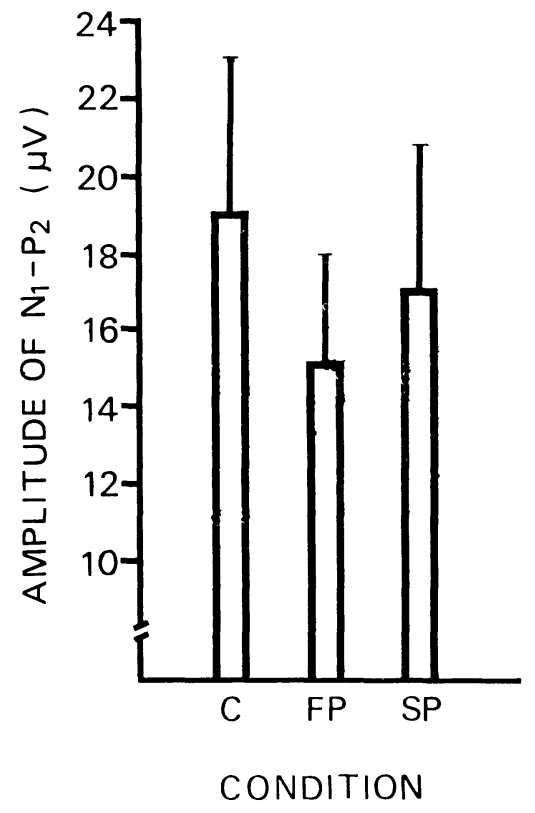

Figure 4. The mean amplitudes of the N1-P2 component for 11 subjects under three conditions. The value in the FP condition is clearly smaller than those in the other conditions.

$t$ test revealed a significant difference between the $\mathrm{C}$ and FP conditions $[\mathrm{t}(10)=3.39, \mathrm{p}<.01]$ and also a significant difference between the SP and FP conditions $[t(10)=3.34, p<.01]$; no significant difference was found between the $\mathrm{C}$ and SP conditions.

\section{Discussion}

The analysis of the N1-P2 component revealed a significant difference between the SP and FP conditions, although, within the 45-msec duration, both conditions were equal in their temporally integrated energies. This difference cannot be attributed to a difference in rise time, since many studies have shown that N1-P2 amplitudes are greater for the sound with a fast rise time than they are for the sound with a slow one (Lamb \& Graham, 1967; Onishi \& Davis, 1968; Ruhm \& Jansen, 1969; Skinner \& Jones, 1968). It would appear that the difference of the N1-P2 amplitudes between the SP and FP conditions is due largely to the temporal location of the plateau intensity, so this finding seems to support the hypothesis that the stimulus intensity around $30 \mathrm{msec}$ after the stimulus onset is more effective for evoking the N1-P2 component than is the intensity immediately after the stimulus onset. Furthermore, there was no significant difference between the $\mathrm{C}$ and SP conditions for the N1-P2 component. This finding piovides evidence for the view that this component would not be greatly affected by the simple temporal integration of the stimulus energy and supports the hypothesis stated above, since maximum intensities in both conditions existed around $30 \mathrm{msec}$ after the onset. The significant difference between the $\mathrm{C}$ and FP conditions for the N1-P2 component is also interpretable by the temporal location effect of the stimulus intensity. In this way, the N1-P2 component seems to be most strongly affected by the stimulus intensity around $30 \mathrm{msec}$ after stimulus onset. If the stimulus duration was less than $30 \mathrm{msec}$, this component could perhaps be affected by the echoic neural traces which remain in the brain around $30 \mathrm{msec}$ after stimulus onset.

The above interpretations have been discussed with the assumption that the amplitudes of the averaged evoked potentials obtained in this study were related exclusively to the ON-response. Schweitzer (1977), on the other hand, stressed the importance of the OFF-response in auditory evoked potentials, and asserted that the averaged evoked potentials to the auditory stimuli of short duration consisted of overlapping of $\mathrm{ON}$ - and OFF-responses. Spreng (Note 1), also, reported that a sound duration of around $50 \mathrm{msec}$, especially, caused an increase in the amplitude of the N1-P2 component based on the overlapping effect referred to above. Actually, in this experiment the fall times were faster in the $\mathrm{C}$ and SP conditions than in the FP condition. Therefore, the OFF-response might be greater in the first two conditions than in the last one. If the evoked potentials to a short sound are more strongly affected by the OFF-response rather than the ONresponse, the finding in Experiment 1 might be explained in terms of the OFF-response. The next experiment was conducted to explore the effect of the OFF-response by manipulating the fall time at $40 \mathrm{msec}$ after the stimulus onset.

\section{EXPERIMENT 2}

\section{Method}

The subjects were seven college students with normal hearing, between the ages of 18 and 24 years. They were paid for their services.

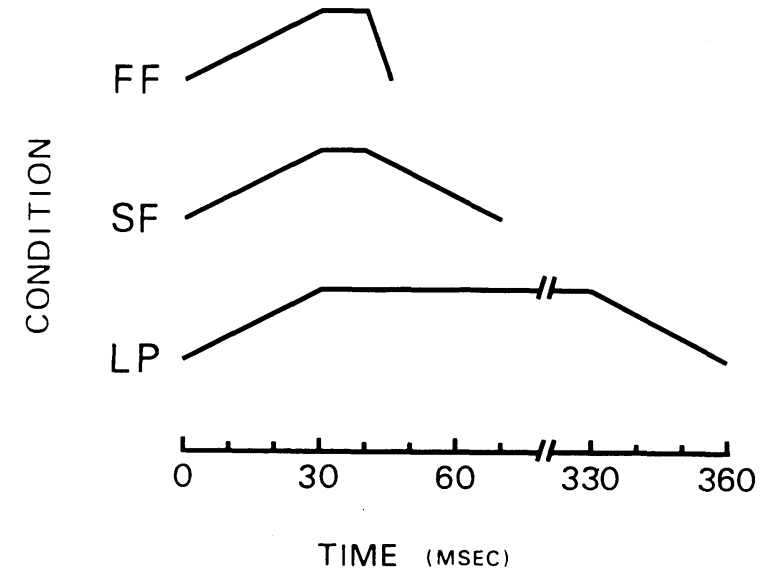

Figure 5. The three types of the shaping-envelopes generated with CPU for noise bursts which differ in the fall time or the plateau duration. 
The apparatus and hearing conditions were the same as those in Experiment 1. Figure 5 shows three types of shaping-envelope for the patterns of noise burst used in Experiment 2. The rise time in all conditions was $30 \mathrm{msec}$. The pattern of the FF condition (top row) was identical to that of Condition SP in Experiment 1 . The SF condition was different from the FF condition only in respect to the fall time. The fall time of the SF condition was $30 \mathrm{msec}$, which was longer than the time used in the FF condition. The LP condition had a much larger plateau duration than the other two conditions. As the fall period in the LP condition began $330 \mathrm{msec}$ after stimulus onset, it was predicted that the OFF-response could not affect the N1-P2 component, whose latency was shorter than $300 \mathrm{msec}$.

After the 15 preliminary trials, the subject was presented randomly with three types of noise burst at a rate of $1 / 2.5 \mathrm{sec}$. Each type of noise burst was presented 50 times. Other recording procedures and brain analyses were the same as in Experiment 1 .

\section{Result}

The typical patterns of the evoked potentials of three subjects are shown in Figure 6. No consistent differences in amplitudes and latencies were observed among the three conditions. Figure 7 presents the mean N1-P2 amplitudes of seven subjects under the three conditions. The N1-P2 amplitudes seem to be largest in the LP condition and smallest in the FF condition. However, a one-factor analysis of variance with repeated measurements showed no significant difference.

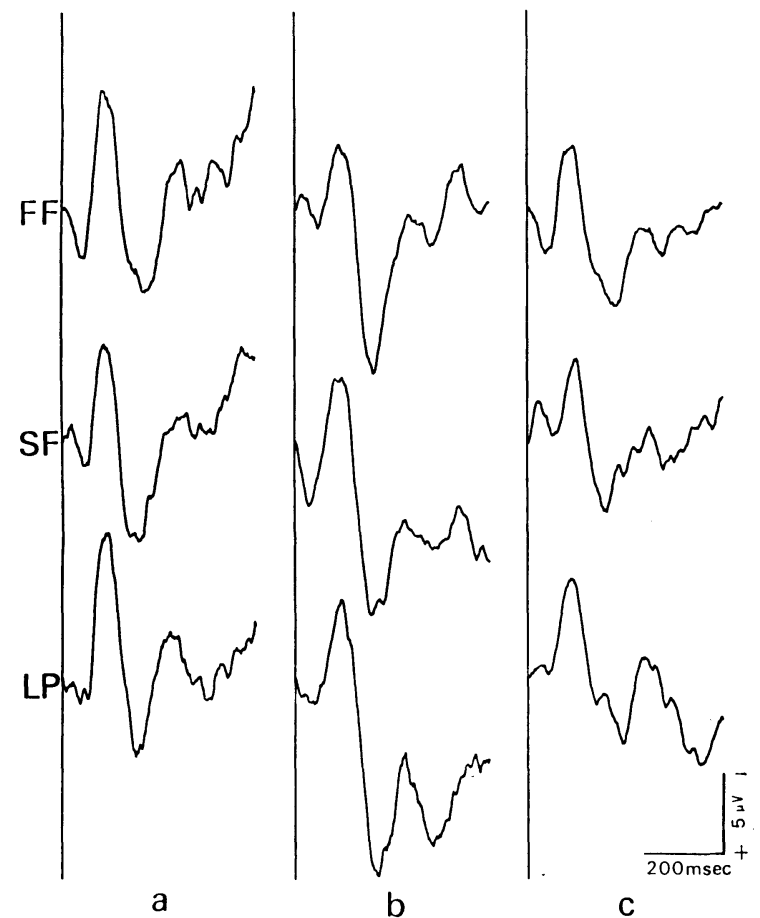

Figure 6. The typical auditory evoked potentials of three subjects under three conditions in Experiment 2.

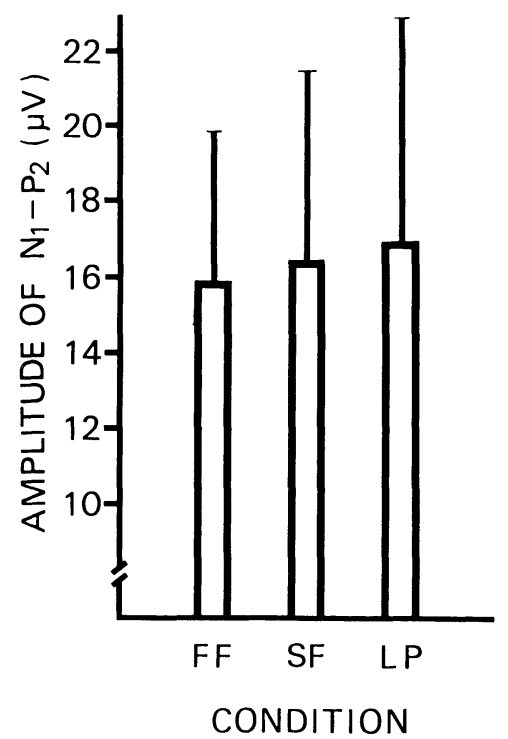

Figure 7. The mean amplitudes of the N1-P2 component for seven subjects under three conditions. There is no great difference among the three conditions.

\section{GENERAL DISCUSSION}

Experiment 2 was undertaken to decide whether the temporal location effect of the plateau intensity obtained in Experiment 1 was appreciably affected by the OFF-response. The FF condition in which fall time was rather fast did not evoke significantly larger amplitudes on the N1-P2 component than did the SF condition, in which the fall time was slow. Furthermore, there was a trend, with the fall period beginning $330 \mathrm{msec}$ after the stimulus onset, for N1-P2 amplitudes to be slightly larger in the LP condition than in the other two conditions. These results would appear to indicate that the OFF-response was not as important as the ON-response in the evoked potentials to a sound with short durations of less than $50 \mathrm{msec}$. This interpretation seems to coincide with the findings of Hillyard and Picton (1978) and Onishi and Davis (1968), who reported that the OFF-response to a brief sound, if present at all, was very small. Thus, the results obtained in Experiment 1 would seem to be attributable not to the effect of the OFF-response but to the temporal location effect of plateau intensity.

It is not yet clear why stimulus intensity around $30 \mathrm{msec}$ after the stimulus onset is so effective in evoking the N1-P2 component. However, it is possible to speculate that masking phenomena might give a clue to the temporal location effect. As generally observed in masking experiments (e.g., Turvey, 1973), in which a stimulus is followed by another in a brief interval, the first stimulus is often masked and only 
the second one is clearly recognizable. Massaro (1977), in an auditory masking experiment, reported that the recognition of sound was heavily deteriorated by backward masking when a mask followed the target within $40 \mathrm{msec}$, but it was not much influenced by forward masking when target followed the mask within $40 \mathrm{msec}$ or so. These phenomena seem to reflect that when the stimuli are given within the minimum sampling intervals specific to the sensory modalities, whether stimulus presentation is continuous, as in this experiment, or discrete, as in the masking experiment, the information that is most available in the higher order central nervous system is involved in the stimulus portion that occurs in several 10 s of milliseconds after the onset of the first stimulus. Given that the minimum sampling interval in the auditory system is $30 \mathrm{msec}$ or so, it is conceivable that the N1-P2 component was most affected by the stimulus intensity around $30 \mathrm{msec}$ after the stimulus onset.

\section{REFERENCE NOTE}

1. Spreng, M. Short sound stimulation and temporal integration model of evoked responses. Paper presented at the Biennial Symposium of the International ERA Study Group, London, 1975.

\section{REFERENCES}

Hillyard, S. A., \& Picton, T. W. ON and OFF components in the auditory evoked potential. Perception \& Psychophysics, 1978, 24, 391-398.
Lamb, L. E., \& Graham, J. T. Influence of signal variables on evoked response to sound. Journal of Speech and Hearing Research, 1967, 10, 257-267.

Massaro, D. W. Capacity limitations in auditory information processing. In S. Dornic (Ed.), Attention and performance. Hillsdale, N.J: Erlbaum, 1977.

McCandless, G. A., \& Best, L. Summed evoked response using pure-tone stimuli. Journal of Speech and Hearing Research, 1966, 9, 266-272.

Olsen, W. O., \& Carhart, R. J. Integration of acoustic power at threshold by normal hearers. Journal of the Acoustical Society of America, 1966, 40, 591-599.

Onishi, S., \& Davis, H. Effects of duration and rise time of tone bursts on evoked V potentials. Journal of the Acoustical Society of America, 1968, 44, 582-591.

Picton, T. W., Woods, D. L., \& Proulx, G. B. Human auditory sustained potentials. 1. The nature of the response. Electroencephalography and Clinical Neurophysiology, 1978, 45, 186-197.

Ruhm, H. B., \& Jansen, J. W. Rate of stimulus change and the evoked response: 1. Signal rise-time. Journal of Auditory Research, 1969, 3, 211-216.

Schwe itze R, P. K. Auditory evoked brain response: Comparison of ON and OFF response at long and short durations. Perception \& Psychophysics, 1977, 22, 87-94.

Skinner, P. H., \& Jones, H. C. Effects of signal duration and rise time on the auditory evoked potential. Journal of Speech and Hearing Research, 1968, 11, 301-306.

TURVEY, M. T. On peripheral and central processes in vision: Inferences from an information-processing analysis of masking with patterned stimuli. Psychological Review, 1973, 80, 1-52.

ZwisLOCKI, J. J. Theory of temporal auditory summation. Journal of the Acoustical Society of America, 1960, 32, 1046-1060.

ZwIsLocki, J. J. Temporal summation of loudness: An analysis. Journal of the Acoustical Society of America, 1969, 46, 431-441.

(Manuscript received November 13, 1979; revision accepted for publication August 18, 1981.) 